\title{
Influência dos fatores estratégia, inovação e tecnologia nos custos da qualidade no arranjo produtivo local de confecções
}

\section{Influence of the strategy, innovation and technology factors on quality costs in clothing industry}

Influencia de los factores de estrategia, innovación y tecnología en los costes de calidad en la industria del vestido

Juliane Andressa Pavão

Mestra em Ciências Contábeis na Universidade Estadual de Maringá (UEM)

Professora do Colegiado de Ciências Contábeis da Universidade Estadual do Paraná

(UNESPAR) Campus de Campo Mourão

Endereço: Av. Comendador Norberto Marcondes, 733 - Centro

CEP: 87302-060, Campo Mourão - PR - Brasil

E-mail: julianepavao@hotmail.com

Telefone: (44) 99706-4881

\section{Reinaldo Rodrigues Camacho}

Doutor em Controladoria e Contabilidade pela Universidade de São Paulo (USP)

Professor do Departamento de Ciências Contábeis da Universidade Estadual de Maringá

(UEM)

Endereço: Av. Colombo, 5790 - Jardim Universitário

CEP: 87020-900, Maringá - PR - Brasil

E-mail: reinaldo.rcamacho@gmail.com

Telefone: (44) 3011-6025

\section{Márcia Maria dos Santos Bortolocci Espejo}

Doutora em Controladoria e Contabilidade pela Universidade de São Paulo (USP)

Professora do Departamento de Ciências Contábeis da Universidade Federal do Mato Grosso do Sul (UFMS)

Endereço: Av. Costa e Silva, s/n - Cidade Universitária

CEP: 79070-900, Campo Grande - MS - Brasil

E-mail: marciabortolocci@gmail.com

Telefone: (67) 3345-7219

\section{Kelly Cristina Mucio Marques}

Doutora em Controladoria e Contabilidade pela Universidade de São Paulo (USP)

Professora do Departamento de Ciências Contábeis da Universidade Estadual de Maringá

(UEM)

Endereço: Av. Colombo, 5790 - Jardim Universitário

CEP: 87020-900, Maringá - PR - Brasil

E-mail: kcmmarques@uem.br

Telefone: (44) 3011-4909

Artigo recebido em 23/10/2016. Revisado por pares em 24/02/2017. Reformulado em 08/03/2018. Recomendado para publicação em 08/03/2018 por Carlos Eduardo Facin Lavarda (Editor-Chefe). Publicado em 28/03/2018. 


\title{
Resumo
}

Este estudo investiga, sob a luz da teoria da contingência, a influência dos fatores contingenciais internos estratégia, tecnologia e inovação na ocorrência dos Custos da Qualidade (CQ). Avaliase também, os efeitos da ocorrência dos CQ no desempenho organizacional. A amostra envolveu 121 empresas do Arranjo Produtivo Local (APL) de confecções nos municípios de Maringá e Cianorte, Estado do Paraná. A estratégia de pesquisa adotada é o levantamento com coleta de dados por meio de questionário, cujos dados foram analisados diante da modelagem de equações estruturais. Os achados apontam que a ocorrência dos CQ não influencia o desempenho organizacional. Porém, a estratégia de diferenciação possui um efeito de redução na ocorrência dos custos de falhas internas e externas, além da estratégia de liderança em custos que incentiva que os custos com avaliação ocorram e restringe a ocorrência de custos com falhas tanto internas como externas. Constatou-se que a adoção de tecnologia de informação auxilia a ocorrência de custos de prevenção e a reduzir custos de falhas internas, bem como, a utilização de tecnologia de produção reduz os custos com devoluções e substituições de produtos. E ainda, a inovação dos processos apresentou um efeito negativo sobre os custos de falhas internas e externas.

Palavras-chave: Custos da Qualidade; Teoria da Contingência; Indústria de confecções; Controle gerencial

\begin{abstract}
This study investigates, under the light of contingency theory, the influence of internal contingency factors, strategy, technology and innovation on the occurrence of Quality Costs (QC). It is also evaluated the effects of the occurrence of QCs on organizational performance. The sample involved 121 from the clothing cluster companies in the counties of Maringá and Cianorte, State of Paraná. The research strategy adopted was the survey with data collection through a questionnaire, whose data were analyzed before the modeling of structural equations. The findings indicate that the occurrence of QCs do not influence organizational performance. However, the differentiation strategy has a reduction effect on the occurrence of internal and external failure costs, as well as the cost leadership strategy that encourages valuation costs to occur and restricts the occurrence of costs with both internal and external failures. It was found that the adoption of information technology helps prevent the occurrence of prevention costs and reduces costs of internal failures, as well as the use of production technology reduces costs with product returns and substitutions. In addition, process innovation has had a negative effect on the costs of internal and external failures.
\end{abstract}

Keywords: Quality costs; Contingency Theory; Clothing industry; Management control

\section{Resumen}

Este estudio investiga, bajo la luz de la teoría de la contingencia, la influencia de los factores contingentes internos estrategia, tecnología e innovación en la ocurrencia de los Costos de la Calidad (CQ). Se evalúa también, los efectos de la ocurrencia de los CQ en el desempeño organizacional. La muestra involucró a 121 empresas del Arreglo Productivo Local (APL) de confecciones en los municipios de Maringá y Cianorte, Estado de Paraná. La estrategia de investigación adoptada es el levantamiento con recolección de datos por medio de cuestionario, cuyos datos fueron analizados ante el modelado de ecuaciones estructurales. Los hallazgos apuntan que la ocurrencia de los CQ no influencia el desempeño organizacional. Sin embargo, la estrategia de diferenciación tiene un efecto de reducción en la ocurrencia de los costos de fallas internas y externas, además de la estrategia de liderazgo en costos que incentiva que los costos de evaluación ocurran y restringe la ocurrencia de costos con fallas tanto internas como externas. Se constató que la adopción de tecnología de información ayuda a la aparición de 
costos de prevención ya reducir costos de fallas internas, así como la utilización de tecnología de producción reduce los costos con devoluciones y sustituciones de productos. Además, la innovación de los procesos presentó un efecto negativo sobre los costos de fallas internas y externas.

Palabras claves: Costes de calidad; Teoría de la contingencia; Industria textil; Control de gestión

\section{Introdução}

O setor têxtil e de confecções é o segundo maior empregador da indústria paranaense, representando 6.400 indústrias que geram 94 mil postos de trabalho e pagam $R \$ 1,3$ bilhão em salários por ano (FIEP, 2015). Porém, devido a crise econômica, o setor começou a demitir e envidar ações para reverter este cenário. Os sindicatos de trabalhadores e sindicatos industriais buscam soluções para reduzir o impacto da crise econômica e evitar novas demissões (FIEP, 2015).

A produção física do setor de confecções tem diminuído nos últimos anos, ao mesmo tempo em que as importações estão aumentando. Para superar a crise e o aumento das importações, o setor de confecções aposta na qualidade e na boa recepção dos compradores varejistas de todas as regiões do país para vencer as dificuldades (AYRES, 2012).

A gestão dos Custos da Qualidade (CQ) permite fabricar produtos com alta qualidade ao menor custo possível (SAKURAI, 1997), já que os custos caem devido à menor quantidade de erros, atrasos, defeitos e reparos, aumentando a produtividade e a fatia de mercado por fornecer produtos de melhor qualidade e preços menores (DEMING, 2003).

Fatores como o posicionamento estratégico das empresas (BAINES; LANGFIELDSMITH, 2003), o investimento em tecnologias de informação e de produção (BAINES; LANGFIELD-SMITH, 2003; HYVÖNEN, 2007) e ainda, a inovação (COSTA; ROCHA, 2009; SERRÃO, 2009) dos produtos e processos podem auxiliar na qualidade dos produtos (CORAL, 1996), contribuindo consequentemente com o desempenho organizacional.

A gestão dos CQ em ambientes competitivos é fator preponderante para melhoria de desempenho (CORAL, 1996), já que os clientes buscam por melhor qualidade a menores preços devido à abertura de mercados em que produtos concorrem entre si. Diante de um cenário de crise econômica e crescimento das importações, é necessária uma mudança no posicionamento das empresas de confecções para se manterem competitivas no mercado, uma vez que fatores externos e internos influenciam no contexto organizacional. Assim, o problema a ser investigado é: Qual a influência dos fatores contingentes estratégia, tecnologia e inovação na ocorrência dos CQ, e ainda, a influência da ocorrência dos CQ no desempenho organizacional de empresas pertencentes ao Arranjo Produtivo Local (APL) de confecções?

A Teoria da Contingência tem norteado estudos relativos ao controle gerencial (AGUIAR; FREZATTI, 2007; CADEZ; GUILDING, 2008; CHENHALL, 2003; GUERRA, 2007; JUNQUEIRA, 2010). Segundo essa abordagem, não há uma estrutura organizacional única que seja efetiva em todas as organizações, mas sim que a otimização da estrutura se modificará de acordo com determinados fatores e, portanto, a organização é vista adaptando-se ao ambiente (DONALDSON, 2001). Desse modo, este estudo tem como objetivo investigar a influência dos fatores contingenciais internos estratégia, tecnologia e inovação na ocorrência dos custos da qualidade, além dos efeitos da ocorrência dos custos da qualidade sobreo desempenho percebido das organizações pertencentes ao APL de confecções dos municípios de Maringá e Cianorte.

Esta pesquisa se justifica devido à importância da qualidade para evitar produtos com defeitos, sobras de materiais, retrabalhos, entre outras falhas, a fim de otimizar o desempenho 
das empresas pertencentes ao APL de confecções. Como também, a dependência econômica do setor de confecções pelos municípios estudados, já que contribui significativamente na geração de emprego e renda, sendo importante no contexto econômico e social do Paraná (OLIVEIRA; CÂMARA; BAPTISTA, 2007).

De modo teórico, este estudo contribui para academia por adicionar conhecimento sobre um tema pouco explorado na área de contabilidade, os custos da qualidade (PINTO, 2012), evidenciando os fatores que podem influenciar esses custos e o desempenho. A contribuição prática consiste em difundir a gestão dos CQ nas empresas de confecções, além de gerar informações para as organizações pertencentes ao APL no que se refere à estratégia, tecnologia e inovação em busca de melhorar a qualidade e o desempenho frente a um ambiente de crise e crescimento de importações.

\section{Framework e hipóteses teóricas da pesquisa}

Os Custos da Qualidade (CQ) são baseados na premissa implícita de que os defeitos têm um custo. Assim, em 1951 Joseph Juran abordou em seu livro Quality Control Handbook, que os custos para atingir a qualidade podiam ser divididos em custos evitáveis, associados à prevenção e custos inevitáveis, relacionados aos defeitos e falhas (GARVIN, 2002).

Feigenbaum (1994) classifica os CQ em custos do controle e custos provenientes de falhas no controle. Os custos do controle são divididos em duas categorias, custos de prevenção, que impedem as não-conformidades e compreendem os gastos com a qualidade; e os custos de avaliação, que abrangem custos de manutenção da qualidade da organização por meio de análises formais dos produtos.

Os custos provenientes de falhas no controle são causados por materiais e produtos que não atendem às especificações, são divididos em custos de falhas internas, que incluem custos dentro da organização, como material refugado, danificado e retrabalhado; e custos das falhas externas, que incluem custos situados na parte externa da organização, como falhas provenientes do desempenho do produto e reclamações dos clientes (FEIGENBAUM, 1994).

Juran e Gryna (1970) afirmam que não existem duas organizações iguais, porém há semelhanças suficientes entre elas que permitem a generalização das categorias dos CQ, sendo que alguns custos podem incorrer em uma organização e não em outra. Dessa forma, são demonstrados na Tabela 1 alguns exemplos de custos ou atividades destas quatro classificações de CQ, com base em pesquisas como Juran e Gryna (1970), Feigenbaum (1994), Robles (2003) e Hansen e Mowen (2010).

Como exemplo de custos de prevenção pode-se citar a manutenção preventiva dos equipamentos, treinamento de pessoal, desenvolvimento de projetos de produtos, desenvolvimento e auditoria de sistemas de qualidade. Já alguns dos custos de avaliação são testes e inspeções nos materiais comprados, testes e inspeções nos produtos fabricados, supervisão e custo da área de inspeção, depreciação dos equipamentos de testes e avaliação da deterioração das matérias primas (JURAN; GRYNA, 1970; FEIGENBAUM, 1994; ROBLES, 2003; HANSEN; MOWEN, 2010).

Com relação aos custos de falhas internas tem-se o retrabalho, redesenho, refugos e sucatas, tempo perdido devido a compra de materiais defeituosos e inspeção em lotes retrabalhados. No que se refere aos custos de falhas externas menciona-se os custos com refaturamento, vendas perdidas devido ao mau desempenho de assistência técnica, devolução de vendas e custos do departamento de assistência técnica (JURAN; GRYNA, 1970; FEIGENBAUM, 1994; ROBLES, 2003; HANSEN; MOWEN, 2010).

Com a gestão dos CQ, os custos de prevenção aumentam e ocorre a redução no número de defeitos e não-conformidades no produto, reduzindo assim, os custos das falhas. Esse mesmo 
aumento nos custos de prevenção tem efeito positivo sobre os custos de avaliação, sendo necessárias menos atividades de inspeção (FEIGENBAUM, 1994).

Assim, fatores externos e internos à organização podem influenciar na gestão e mensuração dos CQ. Nesse sentido, a Teoria da Contingência adere aos princípios da abordagem sistêmica, enfatizando as organizações como sistemas abertos, sendo que as variáveis internas são influenciadas pelo ambiente, de forma independente (ESPEJO, 2008). Além disso, essas variáveis influenciam na estrutura organizacional e consequentemente no desempenho organizacional. Sendo assim, à luz dos fatores contingenciais estratégia, tecnologia e inovação, o framework usado nessa pesquisa é apresentado na Figura 1.

Figura 1 - Modelo estrutural teórico da pesquisa

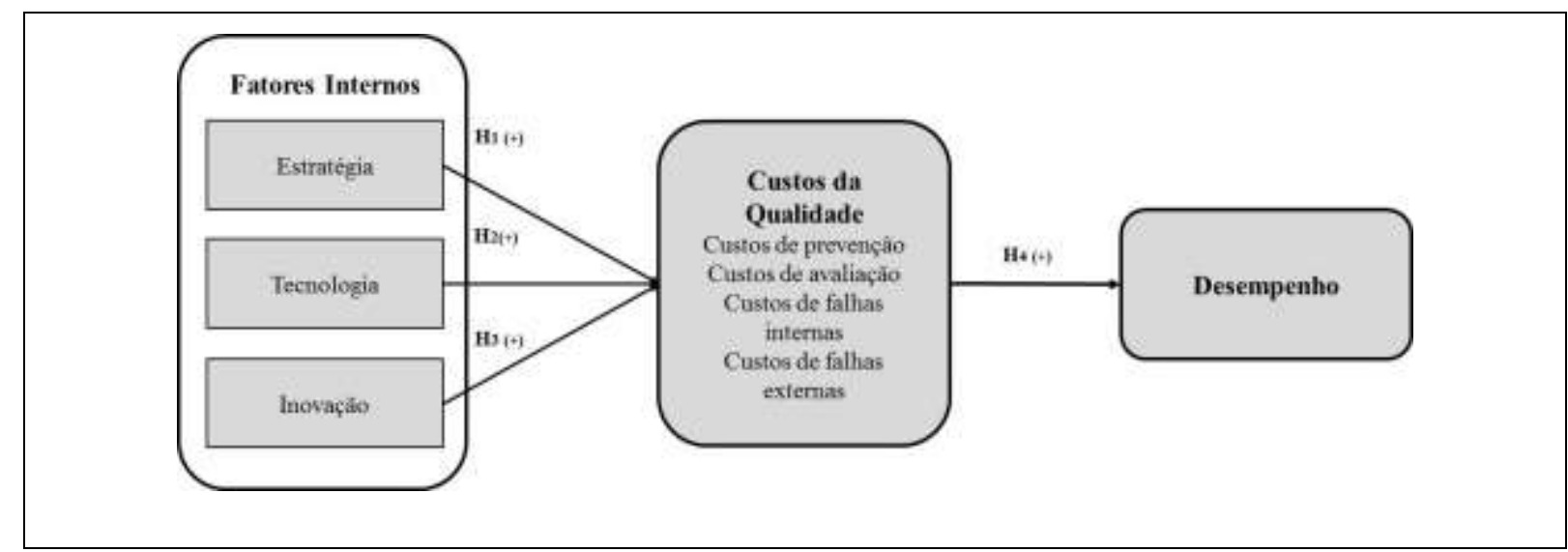

Fonte: Elaborado pelos autores.

Quanto ao fator contingencial estratégia, Langfield-Smith (1997) afirma que o conhecimento sobre a relação entre o Sistema de Controle Gerencial (SCG) e a estratégia é limitado, proporcionando uma margem considerável para futuras investigações. Assim, esta pesquisa investiga a estratégia, um "fator contingencial interno controlável pela empresa" (ESPEJO, 2008, p. 67), para verificar sua influência nos CQ e no desempenho.

Segundo Porter (1996), a essência do posicionamento estratégico é escolher atividades que sejam diferentes daquelas das concorrentes, ou seja, significa realizar atividades diferentes, ou ainda, realizar atividades semelhantes, mas de formas diferentes (PORTER, 1996). As estratégias genéricas se referem às opções estratégicas que as organizações adotam e que orientam todo o seu processo de gestão com o objetivo de enfrentar as forças competitivas e são classificadas em [i] liderança de custo e [ii] diferenciação (PORTER, 2004).

A estratégia liderança de custo total ou estratégia de baixo custo é adotada por empresas que buscam a liderança competitiva por meio de um conjunto de políticas funcionais orientadas para a produção e distribuição de produtos ou serviços a custos inferiores aos seus concorrentes (PORTER, 2004; JOHNSON; SCHOLES; WHITTINGTON, 2007). Por outro lado, a estratégia de diferenciação é seguida pela organização que compete por meio da produção e distribuição de produtos ou serviços que sejam percebidos pelos seus clientes como sendo únicos e diferentes daqueles oferecidos pelos concorrentes (JOHNSON; SCHOLES; WHITTINGTON, 2007).

Segundo o estudo de Baines e Langfield-Smith (2003), a estratégia de diferenciação pode resultar no uso de práticas contábeis de gestão. Pode-se perceber que o tipo de estratégia adotada por uma organização direciona ações a fim de ofertar produtos diferenciados com alta qualidade e/ou baixo custo, sendo que a gestão dos CQ propõe melhorar a qualidade por meio da redução de custos com falhas internas e externas (ROBLES, 2003). Com base nesses argumentos, elabora-se a hipótese 1. 


\section{H1: A estratégia influencia os custos da qualidade.}

O fator tecnologia, para Chenhall (2003) refere-se à forma que uma organização opera, ou seja, como ela transforma insumos em produtos, e neste procedimento inclui hardware (como máquinas e ferramentas), materiais, pessoas, software e conhecimento. A tecnologia pode ser dividida em duas dimensões, a tecnologia da informação e a tecnologia associada às práticas de manufatura (BURNS; VAIVIO, 2001; CHENHALL, 2003; HYVÖNEN, 2007).

Burns e Vaivio (2001) afirmam que avanços na tecnologia da informação têm impulsionado a inovação e a mudança na coleta, mensuração, análise e comunicação das informações dentro e entre as organizações. Desse modo, no estudo de Hyvönen (2007) a tecnologia da informação se refere à ênfase da empresa em aplicações avançadas de sistemas de informação e utilizou para medir somente variáveis que representam a utilização de inovações de tecnologia da informação em geral. Baines e Langfield-Smith (2003) mencionam que a implementação de tecnologia de fabricação avançada é uma forma em que organizações podem responder às crescentes solicitações de clientes por qualidade, flexibilidade e confiabilidade do fornecimento de produtos.

Neste sentido, assumindo as considerações de que a tecnologia pode auxiliar as organizações atenderem as solicitações de produtos com qualidade por parte dos clientes (BAINES; LANGFIELD-SMITH, 2003), a hipótese 2 busca testar se a tecnologia de informação e de produção afeta a qualidade dos produtos.

\section{H2: A tecnologia influencia os custos da qualidade.}

A inovação faz a diferença em todas as organizações. É necessário alterar os produtos e serviços que são ofertados para não correr o risco de serem superados pela concorrência (BESSANT; TIDD, 2009). Segundo Van de Ven (1986), a inovação pode ser compreendida como o desenvolvimento e implementação de novas ideias por pessoas ao longo do tempo e é voltada para quatro fatores básicos: novas ideias, pessoas, transações e contexto institucional.

A administração das organizações está começando a perceber que a inovação gera vantagens duradouras e produz mudanças no posicionamento competitivo (DOBNI, 2008), sendo essencial para a sobrevivência organizacional num mercado concorrente (SERRÃO, 2009). A introdução de novas ideias conduz a melhorias organizacionais significativas em termos de novos produtos ou processos internos, o que contribui com o desempenho organizacional (SERRÃO, 2009). Assim, as inovações contribuem nas questões de como manter ou ampliar o mercado, na melhoria da qualidade do produto e no aumento da capacidade e flexibilidade produtiva (COSTA; ROCHA, 2009). Por conseguinte, sugere-se que o grau de inovação dos produtos e processos implica nos custos da qualidade, tal qual a hipótese 3.

\section{H3: A inovação influencia os custos da qualidade}

Por fim, em estudos que se classificam como de abordagem contingencial de sistemas (DRAZIN; VAN DE VEN, 1985) é necessária a mensuração do desempenho para relacionar essa variável com outras variáveis internas e externas à organização. Destarte, foram selecionados indicadores de mensuração do desempenho relacionados aos custos da qualidade e a organização. Quanto ao desempenho dos custos da qualidade, verificou-se o grau de satisfação ao auxiliar na redução dos custos, na melhoria dos processos, na satisfação dos clientes, no gerenciamento da empresa e na tomada de decisão (ESPEJO, 2008; NEITZKE, 2015).

Existem diferentes formas de mensurar o desempenho organizacional, como o retorno sobre o patrimônio e o retorno sobre o investimento (ESPEJO, 2008), como também uma autoavaliação de desempenho em comparação com os concorrentes (GUERRA, 2007; JUNQUEIRA, 2010). Para esta pesquisa optou-se pelo desempenho percebido pelo gestor (NEITZKE, 2015), conforme suas expectativas com relação ao volume de vendas, à margem de lucro, ao retorno do investimento e o desempenho de modo geral. 
Verificou-se a influência da ocorrência dos custos da qualidade no desempenho das organizações. Uma vez que os produtos estão sendo produzidos corretamente pela primeira vez e em conformidade com seus requisitos, não ocorrendo defeitos, reparos, sobras de materiais, descontos em vendas de produtos com pequenos defeitos (FEIGENBAUM, 1994; ROBLES, 2003; JURAN, 2009), os custos de falhas internas e externas são reduzidos ou eliminados podendo resultar na otimização do resultado, e ainda segundo Coral (1996) a falta de qualidade impacta negativamente na lucratividade e no desempenho. Desse modo, formula-se então a última hipótese a ser testada:

\section{H4: Os custos da qualidade influenciam o desempenho.}

\section{Estratégia Metodológica}

A presente pesquisa possui caráter epistemológico positivista, quantitativo e descritivo, a partir de um levantamento de dados transversal por meio de questionário. A população é constituída por 252 empresas industriais de confecção dos municípios de Maringá e Cianorte, no Estado do Paraná. Foi obtido um total de 121 respostas, sendo que o número de respondentes atingiu o tamanho da amostra mínima com erro amostral de $10 \%$ para os dois municípios investigados. $\mathrm{O}$ questionário é formado por escalas numéricas com intensidade de 11 pontos (nota de 0 a 10) e foi dividido em blocos para aumentar a compreensão do tema das questões. Os três primeiros blocos abordam os fatores contingenciais estratégia, tecnologia e inovação respectivamente. O bloco 4 questiona a ocorrência dos CQ. O quinto bloco interroga sobre o desempenho organizacional percebido pelo gestor. Termina com perguntas que caracterizam o perfil da organização e do respondente. Os constructos delimitados estão expressos na Tabela 1 , bem como sua correspondência nas questões e indicadores do questionário e as referências suporte.

Tabela 1 - Constructos e variáveis da pesquisa

\begin{tabular}{|c|c|c|c|c|}
\hline $\begin{array}{l}\text { Constructos de } \\
1^{\mathrm{a}} \text { ordem }\end{array}$ & $\begin{array}{c}\text { Constructos de } 2^{\mathrm{a}} \\
\text { ordem }\end{array}$ & Constructos latentes & Questões & Referências \\
\hline \multirow{6}{*}{$\begin{array}{c}\text { Fatores } \\
\text { Contingenciais }\end{array}$} & \multirow[b]{2}{*}{ Estratégia } & Diferenciação & \multirow[b]{2}{*}{1} & \multirow{2}{*}{$\begin{array}{c}\text { Gordon e Narayanan (1984), } \\
\text { Hansen e Van der Stede (2004), } \\
\text { Porter (2004) e Espejo (2008). }\end{array}$} \\
\hline & & Custo & & \\
\hline & \multirow{2}{*}{ Tecnologia } & $\begin{array}{l}\text { Tecnologia de } \\
\text { informação }\end{array}$ & 2 & \multirow{2}{*}{$\begin{array}{l}\text { Hansen e Van der Stede (2004), } \\
\text { Hyvönen (2007) e Espejo (2008). }\end{array}$} \\
\hline & & $\begin{array}{c}\text { Tecnologia da } \\
\text { produção }\end{array}$ & 3 & \\
\hline & \multirow{2}{*}{ Inovação } & Inovação de produtos & \multirow{2}{*}{4,5} & \multirow{2}{*}{$\begin{array}{l}\text { Tigre (2006), Bessant e Tidd } \\
\text { (2009) e Cruz (2014). }\end{array}$} \\
\hline & & Inovação de processos & & \\
\hline \multirow{4}{*}{$\begin{array}{l}\text { Custos da } \\
\text { qualidade }\end{array}$} & \multirow{4}{*}{ Ocorrência } & Custos de prevenção & \multirow{4}{*}{9} & \multirow{4}{*}{$\begin{array}{l}\text { Robles (2003), Sá (2003) e } \\
\text { Collaziol (2006) }\end{array}$} \\
\hline & & Custos de avaliação & & \\
\hline & & $\begin{array}{c}\text { Custos de falhas } \\
\text { internas }\end{array}$ & & \\
\hline & & $\begin{array}{l}\text { Custos de falhas } \\
\text { externas }\end{array}$ & & \\
\hline Desempenho & \multicolumn{2}{|c|}{ Desempenho organizacional percebido } & 15,16 & $\begin{array}{c}\text { Guerra (2007), Espejo (2008), } \\
\text { Junqueira (2010) e Neitzke (2015) }\end{array}$ \\
\hline
\end{tabular}

Fonte: Elaborado pelos autores.

O período de coleta de dados ocorreu entre os meses de outubro e dezembro de 2015. Primeiramente, entrou-se em contato por telefone com as organizações, visando à apresentação da pesquisa e identificação do respondente, em seguida foi agendada uma visita para aplicação do questionário pessoalmente. 
Foi realizado o pré-teste com especialistas (docentes da contabilidade), e em seguida, com duas empresas que atuam no mesmo setor para verificar a adequação do instrumento com a finalidade da pesquisa. Na análise dos dados utilizou-se estatística descritiva e modelagem de equações estruturais.

O modelo de equações estruturais foi estimado por meio da abordagem baseada na variância ou método de Mínimos Quadrados Parciais (PLS - Partial Least Square) utilizando o software estatístico SmartPLS 3, pelo fato desta abordagem possuir como vantagem a sua robustez, fornecendo uma solução mesmo quando existem problemas que podem impedir uma solução na modelagem de equações estruturais (HAIR et al., 2009). A análise do modelo seguiu os critérios de Hair et al. (2009), Marôco (2010) e Ringle, Silva e Bido (2014).

\section{Análise e Discussão dos Achados}

A análise dos achados será subdividida em [i] modelo de mensuração, [ii] modelo estrutural e [iii] discussão do teste de hipóteses.

\subsection{Modelo de Mensuração}

Primeiramente verificou-se a normalidade dos dados, portanto foram analisadas as medidas de assimetria e curtose. Adotou-se como parâmetros os limites máximos de 3 para a assimetria e 10 para a curtose (MARÔCO, 2010), sendo que os dados das variáveis observadas não ultrapassaram este parâmetro, o que indica a normalidade dos dados.

A seguir foi realizada a análise da validade do modelo de mensuração, composta pelos testes de validade convergente e validade discriminante. A validade convergente ocorre "quando indicadores de um construto específico convergem ou compartilham uma elevada proporção de variância em comum" (HAIR et al., 2009, p. 589) e possui os seguintes indicadores: [i] cargas fatoriais, [ii] variância extraída e [iii] confiabilidade.

Altas cargas fatoriais indicam que os fatores convergem para algum ponto em comum devendo apresentar um valor superior a 0,5 (HAIR et al., 2009). Nesta pesquisa, foi necessário excluir quatro variáveis observáveis que não apresentaram carga fatorial superior a 0,5. São elas e suas respectivas cargas fatoriais: T9 - diversificação das tarefas $(-0,484)$, E7 - preço de venda menor que da concorrência $(-0,019), \mathrm{T} 1$ - vendas pela internet $(0,437)$ e AV3 - avaliação dos produtos dos concorrentes $(0,445)$. Assim, segue-se com as análises de validade a partir do modelo ajustado.

O próximo passo é a verificação da variância extraída (AVE - Average Variance Extracted) que consiste em uma "medida de convergência em um conjunto de itens que representa um construto latente" (HAIR et al., 2009, 589). Uma variância acima de 0,5 representa uma convergência adequada.

A confiabilidade (CR - Composite Reliability) é uma medida de consistência interna das variáveis medidas representando um construto latente sendo considerado acima de $0,7 \mathrm{um}$ bom valor (HAIR et al., 2009). Os dois últimos indicadores de validade convergente são evidenciados na Tabela 2, no qual se verifica que o modelo atende aos critérios de validade convergente, apresentando AVE superior a 0,5 e CR superior a 0,7.

Foi analisado o coeficiente de determinação de Pearson $\left(\mathrm{R}^{2}\right)$ que faz uma avaliação da "variância das variáveis endógenas, que é explicada pelo modelo estrutural" (RINGLE; SILVA; BIDO, 2014, p. 72). Verifica-se que os valores $\mathrm{R}^{2}$ possuem uma explicação entre média e grande do modelo, como também, o Alfa de Cronbach demonstra sua confiabilidade. 
Tabela 2 - Indicadores de validade convergente para o modelo ajustado

\begin{tabular}{|c|c|c|c|c|}
\hline Constructos latentes & AVE & $\mathbf{C R}$ & $\mathbf{R}^{2}$ & $\begin{array}{c}\text { Alfa de } \\
\text { Cronbach }\end{array}$ \\
\hline \multicolumn{5}{|l|}{ Estratégia } \\
\hline Estratégia de diferenciação & 0,553 & 0,831 & - & 0,740 \\
\hline Estratégia de liderança em custo & 0,640 & 0,766 & - & 0,617 \\
\hline \multicolumn{5}{|l|}{ Tecnologia } \\
\hline Tecnologia de informação & 0,618 & 0,890 & - & 0,845 \\
\hline Tecnologia de produção & 0,814 & 0,897 & - & 0,773 \\
\hline \multicolumn{5}{|l|}{ Inovação } \\
\hline Inovação de produtos & 0,535 & 0,773 & - & 0,563 \\
\hline Inovação de processos & 0,721 & 0,886 & - & 0,811 \\
\hline \multicolumn{5}{|l|}{ Custos da Qualidade } \\
\hline Custos de prevenção & 0,574 & 0,870 & 0,331 & 0,813 \\
\hline Custos de avaliação & 0,679 & 0,864 & 0,189 & 0,769 \\
\hline Custos de falhas internas & 0,593 & 0,878 & 0,195 & 0,829 \\
\hline Custos de falhas externas & 0,699 & 0,874 & 0,213 & 0,785 \\
\hline \multicolumn{5}{|l|}{ Desempenho } \\
\hline Desempenho organizacional & 0,807 & 0,944 & 0,224 & 0,920 \\
\hline $\begin{array}{l}\text { Valores referenciais dos indicadores } \\
\text { de validade convergente }\end{array}$ & $>0,5$ & $>\mathbf{0 , 7}$ & $\begin{array}{l}2 \% \text { (pequeno), } \\
13 \% \text { (médio) e } \\
26 \% \text { (grande) }\end{array}$ & $>0,7$ \\
\hline
\end{tabular}

Fonte: Elaborado pelos autores.

$\mathrm{Na}$ sequência, é averiguada a validade discriminante que representa "quando um constructo é verdadeiramente distinto de outros" (HAIR et al., 2009, p. 589). São comparadas as raízes quadradas dos valores das variâncias extraídas de cada constructo com as correlações de Pearson entre os constructos, sendo que as raízes quadradas das variâncias extraídas devem ser maiores que as correlações dos constructos (RINGLE; SILVA; BIDO, 2014). Os resultados são visualizados na Tabela 3 .

Tabela 3 - Validade discriminante

\begin{tabular}{|c|c|c|c|c|c|c|c|c|c|c|c|}
\hline & AV & F. EX & F. IN & PR & EC & ED & I. Prod. & DO & I. Proc. & TI & TP \\
\hline $\mathbf{A V}$ & 0,824 & & & & & & & & & & \\
\hline F. EX & 0,129 & 0,836 & & & & & & & & & \\
\hline F. IN & 0,129 & 0,665 & 0,770 & & & & & & & & \\
\hline PR & 0,476 & $-0,109$ & $-0,171$ & 0,758 & & & & & & & \\
\hline EC & 0,376 & 0,191 & 0,104 & 0,280 & 0,800 & & & & & & \\
\hline ED & 0,300 & 0,199 & 0,128 & 0,273 & 0,341 & 0,744 & & & & & \\
\hline I. Prod. & 0,170 & $-0,040$ & $-0,080$ & 0,279 & 0,118 & 0,376 & 0,731 & & & & \\
\hline DO & 0,053 & $-0,155$ & $-0,191$ & 0,185 & 0,102 & 0,180 & 0,406 & 0,899 & & & \\
\hline I. Proc. & 0,280 & $-0,147$ & $-0,244$ & 0,413 & 0,445 & 0,363 & 0,583 & 0,398 & 0,849 & & \\
\hline TI & 0,227 & $-0,074$ & $-0,158$ & 0,532 & 0,265 & 0,414 & 0,349 & 0,205 & 0,443 & 0,786 & \\
\hline TP & 0,133 & 0,244 & 0,146 & 0,124 & 0,110 & 0,124 & 0,115 & $-0,009$ & 0,098 & 0,085 & 0,902 \\
\hline
\end{tabular}

Nota: AV - Custos de avaliação; F. EX - Custos de falhas externas; F. IN - Custos de falhas internas; PR - Custos de prevenção; EC - Estratégia de liderança em custo; ED - Estratégia de diferenciação; I. Prod. - Inovação de produtos; DO - Desempenho Organizacional; I. Proc. - Inovação de processos; TI - Tecnologia de informação; TP - Tecnologia de produção.

Fonte: Elaborado pelos autores.

Percebe-se que o modelo atende aos critérios de validade discriminante, pois as raízes quadradas das variâncias extraídas são maiores que as correlações dos constructos. 


\subsection{Modelo estrutural}

O módulo Bootstrapping (técnica de reamostragem) foi utilizado para testar a significância das influências entre os constructos por meio do teste t de Student e o módulo Blindfolding para análise de outros dois indicadores de qualidade de ajuste do modelo: [i] Validade preditiva $\left(\mathrm{Q}^{2}\right)$ ou Indicador de Stone-Geisser e [ii] Tamanho do efeito $\left(\mathrm{f}^{2}\right)$ ou Indicador de Cohen.

$\mathrm{O}$ indicador de validade preditiva avalia quanto o modelo se aproxima do que se esperava dele ou a qualidade da predição do modelo e acurácia do modelo ajustado (RINGLE; SILVA; BIDO, 2014). Já o tamanho do efeito avalia a utilidade de cada constructo para o ajuste do modelo (RINGLE; SILVA; BIDO, 2014). A Tabela 4 evidencia os resultados destes indicadores de qualidade do ajuste, bem como, os valores referenciais.

Tabela 4 - Validade preditiva $\left(Q^{2}\right)$ e tamanho do efeito $\left(f^{2}\right)$

\begin{tabular}{l|c|c}
\hline \multicolumn{1}{c|}{ Constructos latentes } & $\mathbf{Q}^{\mathbf{2}}$ & $\mathbf{f}^{\mathbf{2}}$ \\
\hline Estratégia & - & 0,249 \\
\hline Estratégia de diferenciação & - & 0,138 \\
\hline Estratégia de liderança em custo & - & 0,415 \\
\hline Tecnologia & - & 0,388 \\
\hline Tecnologia de informação & & 0,103 \\
\hline Tecnologia de produção & - & 0,421 \\
\hline Inovação & - & 0,359 \\
\hline Inovação de produtos & & 0,350 \\
\hline Inovação de processos & 0,163 & 0,391 \\
\hline Custos da Qualidade & 0,088 & 0,388 \\
\hline Custos de prevenção & 0,092 & \\
\hline Custos de avaliação & 0,114 & 0,661 \\
\hline Custos de falhas internas & & $\mathbf{0}$ \\
\hline Custos de falhas externas & 0,143 & $\mathbf{0 , 0 2 , 0 , 1 5}$ e 0,35 são pequenos, \\
\hline Desempenho & $\mathbf{Q}^{\mathbf{2}}>\mathbf{0}$ & médios e grandes. \\
\hline Desempenho organizacional & &
\end{tabular}

Fonte: Elaborado pelos autores.

Os dados da Tabela 4 indicam a qualidade do modelo por apresentar a validade preditiva maior que zero e o indicador tamanho do efeito também se mostrou satisfatório com um grande efeito para o ajuste do modelo pela maioria dos constructos.

O software SmartPLS oferece o índice de ajustamento Standardized Root Mean Square Residual (SRMR) que permite avaliar a magnitude média da diferença entre as correlações observadas e esperadas como uma medida de critério de ajuste, sendo valores menores que 0,10 considerados um bom ajuste (SMARTPLS, 2016). O modelo ajustado apresentou SRMR de 0,074 o que é considerado um bom ajustamento.

Sendo assim, a última etapa da avaliação do modelo estrutural consiste na análise dos Path Coefficients, Sample Mean, Desvio Padrão (STDEV), T statistic e p-values, que são apresentados na Tabela 5. 
Tabela 5 - Resultados dos testes

\begin{tabular}{|c|c|c|c|c|c|c|c|}
\hline & Hipóteses & $\begin{array}{l}\text { Efeito } \\
\text { Direto }\end{array}$ & $\begin{array}{c}\text { Sample } \\
\text { Mean }\end{array}$ & STDEV & $\begin{array}{c}\mathbf{T} \\
\text { Statistics }\end{array}$ & $\begin{array}{c}\mathbf{P} \\
\text { Values }\end{array}$ & Resultados \\
\hline H1a & Est. Diferenciação -> C. Prevenção & $-0,008$ & $-0,005$ & 0,101 & 0,077 & 0,939 & Rejeitada \\
\hline $\mathrm{H} 1 \mathrm{~b}$ & Est. Diferenciação -> C. Avaliação & 0,145 & 0,160 & 0,123 & 1,183 & 0,237 & Rejeitada \\
\hline H1c & Est. Diferenciação -> C. F. Internas & 0,216 & 0,225 & 0,105 & $2,054 *$ & 0,040 & Não rejeitada \\
\hline H1d & Est. Diferenciação -> C. F. Externas & 0,237 & 0,253 & 0,118 & $2,016^{*}$ & 0,044 & Não rejeitada \\
\hline $\mathrm{H} 1 \mathrm{e}$ & Est. Custo -> C. Prevenção & 0,083 & 0,082 & 0,081 & 1,023 & 0,307 & Rejeitada \\
\hline H1f & Est. Custo -> C. Avaliação & 0,274 & 0,274 & 0,093 & $2,961 *$ & 0,003 & Não rejeitada \\
\hline $\mathrm{H} 1 \mathrm{~g}$ & Est. Custo $->$ C. F. Internas & 0,237 & 0,236 & 0,112 & $2,105^{*}$ & 0,035 & Não rejeitada \\
\hline $\mathrm{H} 1 \mathrm{~h}$ & Est. Custo $->$ C. F. Externas & 0,270 & 0,262 & 0,119 & $2,270^{*}$ & 0,023 & Não rejeitada \\
\hline $\mathrm{H} 2 \mathrm{a}$ & Tec. Informação -> C. Prevenção & 0,425 & 0,433 & 0,103 & $4,129 *$ & 0,000 & Não rejeitada \\
\hline $\mathrm{H} 2 \mathrm{~b}$ & Tec. Informação -> C. Avaliação & 0,054 & 0,061 & 0,088 & 0,608 & 0,543 & Rejeitada \\
\hline $\mathrm{H} 2 \mathrm{c}$ & Tec. Informação -> C. F. Internas & $-0,169$ & $-0,174$ & 0,082 & $2,057 *$ & 0,040 & Não rejeitada \\
\hline $\mathrm{H} 2 \mathrm{~d}$ & Tec. Informação -> C. F. Externas & $-0,128$ & $-0,130$ & 0,069 & 1,837 & 0,067 & Rejeitada \\
\hline $\mathrm{H} 2 \mathrm{e}$ & Tec. Produção -> C. Prevenção & 0,061 & 0,060 & 0,096 & 0,638 & 0,524 & Rejeitada \\
\hline $\mathrm{H} 2 \mathrm{f}$ & Tec. Produção -> C. Avaliação & 0,072 & 0,074 & 0,096 & 0,745 & 0,456 & Rejeitada \\
\hline $\mathrm{H} 2 \mathrm{~g}$ & Tec. Produção -> C. F. Internas & 0,137 & 0,141 & 0,107 & 1,279 & 0,201 & Rejeitada \\
\hline $\mathrm{H} 2 \mathrm{~h}$ & Tec. Produção -> C. F. Externas & 0,223 & 0,220 & 0,103 & $2,170^{*}$ & 0,030 & Não rejeitada \\
\hline $\mathrm{H} 3 \mathrm{a}$ & In. Produtos -> C. Prevenção & 0,013 & 0,029 & 0,110 & 0,122 & 0,903 & Rejeitada \\
\hline $\mathrm{H} 3 \mathrm{~b}$ & In. Produtos -> C. Avaliação & 0,019 & 0,018 & 0,144 & 0,132 & 0,895 & Rejeitada \\
\hline $\mathrm{H} 3 \mathrm{c}$ & In. Produtos $->$ C. F. Internas & 0,102 & 0,091 & 0,113 & 0,903 & 0,367 & Rejeitada \\
\hline $\mathrm{H} 3 \mathrm{~d}$ & In. Produtos $->$ C. F. Externas & 0,066 & 0,048 & 0,095 & 0,702 & 0,483 & Rejeitada \\
\hline $\mathrm{H} 3 \mathrm{e}$ & In. Processos -> C. Prevenção & 0,177 & 0,163 & 0,128 & 1,381 & 0,168 & Rejeitada \\
\hline $\mathrm{H} 3 \mathrm{f}$ & In. Processos -> C. Avaliação & 0,063 & 0,049 & 0,126 & 0,501 & 0,616 & Rejeitada \\
\hline $\mathrm{H} 3 \mathrm{~g}$ & In. Processos -> C. F. Internas & $-0,427$ & $-0,412$ & 0,112 & $3,823 *$ & 0,000 & Não rejeitada \\
\hline $\mathrm{H} 3 \mathrm{~h}$ & In. Processos -> C. F. Externas & $-0,358$ & $-0,333$ & 0,114 & $3,140 *$ & 0,002 & Não rejeitada \\
\hline $\mathrm{H} 4 \mathrm{a}$ & C. Prevenção -> Desemp. Org. & 0,032 & 0,024 & 0,122 & 0,262 & 0,793 & Rejeitada \\
\hline $\mathrm{H} 4 \mathrm{~b}$ & C. Avaliação -> Desemp. Org. & $-0,054$ & $-0,054$ & 0,116 & 0,468 & 0,640 & Rejeitada \\
\hline $\mathrm{H} 4 \mathrm{c}$ & C. F. Internas -> Desemp. Org. & $-0,070$ & $-0,079$ & 0,100 & 0,701 & 0,484 & Rejeitada \\
\hline $\mathrm{H} 4 \mathrm{~d}$ & C. F. Externas -> Desemp. Org. & $-0,056$ & $-0,043$ & 0,106 & 0,525 & 0,600 & Rejeitada \\
\hline
\end{tabular}

Nota: * é significante a $5 \%$

Fonte: Elaborado pelos autores.

As hipóteses foram avaliadas por meio do teste $\mathrm{t}$ de Student utilizando um nível de significância de $5 \%$ e o valor referencial de $t \geq 1,96$ (RINGLE; SILVA; BIDO, 2014). Na sequência foi feita a discussão do teste de hipóteses.

\subsection{Discussão do teste de hipóteses}

Primeiramente, é interessante mencionar que 52,9\% das empresas investigadas afirmam mensurar algum dos CQ, sendo que $42 \%$ mencionam que a mensuração destes custos é útil, porém existem outras prioridades na empresa. Das empresas que mensuram os CQ, 11\% discriminam estes custos em custos de conformidade e não conformidade e $13 \%$ classificam em quatro categorias, custos de prevenção, avaliação, falhas internas e falhas externas.

A hipótese 1 testa se o fator contingencial estratégia influencia a ocorrência dos CQ devido este fator influenciar no uso de práticas contábeis de gestão (BAINES; LANGFIELDSMITH, 2003), podendo a gestão dos CQ ser uma delas. A hipótese 1 contatou que a estratégia de diferenciação influencia para uma menor ocorrência dos custos de falhas internas e externas; a estratégia de liderança em custos incentiva os custos de avaliação e evita a ocorrência de custos com falhas internas e externas, sendo as hipóteses H1c, H1d, H1f, H1g e H1h suportadas. Esse resultado indica que os dois tipos de estratégia podem influenciar os $C Q$, especialmente 
os custos de falhas internas e falhas externas. Mesmo apresentando objetivos diferentes em cada tipologia estratégica foi encontrado que apenas os custos de avaliação se diferenciam entre ambas.

Sugere-se que as empresas de confecções que possuem estratégia voltada para diferenciação, ao investirem em pesquisas e desenvolvimento de seus produtos, tendem a apresentar uma alta qualidade dos mesmos. Desse modo, tendem a uma redução nos custos de falhas internas e externas, como retrabalhos, sobras, devoluções e vendas perdidas, tal como os resultados reportam.

Pelo fato da estratégia de liderança em custos exigir um controle rígido da ocorrência dos mesmos a fim de sua redução, é possível que as empresas estejam investindo no controle da qualidade por meio de inspeções (avaliações) com objetivo de reduzir as falhas internas e externas, na busca de um custo baixo para os produtos ao invés de investir na prevenção.

A hipótese 2 testa se a tecnologia de informação e tecnologia de produção influenciam na ocorrência dos CQ, admitindo que a tecnologia auxilia as organizações atenderem as solicitações de produtos com qualidade por parte dos clientes (BAINES; LANGFIELDSMITH, 2003) de modo que a tecnologia de informação impulsiona a inovação e a mudança na coleta, mensuração, análise e comunicação das informações (BURNS; VAIVIO, 2001) e a tecnologia de produção auxilia as organizações nas crescentes solicitações de clientes por qualidade, flexibilidade e confiabilidade de produtos (BAINES; LANGFIELD-SMITH, 2003).

As hipóteses $\mathrm{H} 2 \mathrm{a}, \mathrm{H} 2 \mathrm{c}$ e $\mathrm{H} 2 \mathrm{~h}$ suportam a tese de que a tecnologia de informação influencia na ocorrência de custos de prevenção e de falhas internas, como também a tecnologia de produção influencia para redução dos custos de falhas externas. Ou seja, o uso de tecnologia de informação possibilita que o fluxo da informação ocorra de forma eficiente, auxiliando na prevenção e na redução da ocorrência de falhas internas, como por exemplo, retrabalho e atrasos na produção.

No que diz respeito à tecnologia de produção no setor analisado, percebe-se que este fator influencia na qualidade dos produtos, uma vez que foi verificado a não ocorrência de devoluções e trocas devido à má qualidade dos produtos.

A hipótese $\mathrm{H} 3$ testou se a inovação de produtos e processos tinha efeito sobre a ocorrência dos CQ partindo do pressuposto que as inovações contribuem na melhoria da qualidade do produto e no aumento da capacidade e flexibilidade produtiva (COSTA; ROCHA, 2009). Tidd, Bessant e Pavitt (2008) afirmam que a inovação dos produtos afeta a qualidade trazendo para organização maior reputação e valor, já a inovação dos processos melhora a qualidade relativa e reduz os custos.

Ao contrário dos autores mencionados, as hipóteses $\mathrm{H} 3 \mathrm{a}, \mathrm{H} 3 \mathrm{~b}, \mathrm{H} 3 \mathrm{c}$ e $\mathrm{H} 3 \mathrm{~d}$ foram rejeitadas, haja vista que a inovação dos produtos não influenciou nenhuma das categorias dos CQ. Porém, com relação à inovação dos processos, as hipóteses H3g e H3h foram aceitas, uma vez que essa variável exerce um efeito negativo na ocorrência dos custos de falhas internas e externas de aproximadamente $42,7 \%$ e $35,8 \%$ respectivamente, ou seja, a inovação de processos causa custos com retrabalho, sobras, atrasos e devoluções de produtos com defeitos em empresas de confecções, uma vez que à medida que as organizações procuram inovar nos processos produtivos, as tarefas se tornam mais incertas (DONALDSON, 2008), induzindo ao erro e consequentemente aos custos de falhas.

Por último, a hipótese 4, averiguou se os custos de prevenção, avaliação, falhas internas e externas possuem efeito sobre o desempenho organizacional. Segundo Feigenbaum (1994), os custos de falhas internas e externas, uma vez reduzidos ou eliminados, resultam na otimização do resultado. Ainda, Coral (1996) constatou que a falta de qualidade impacta negativamente na lucratividade e no desempenho empresarial. A hipótese 4 foi rejeitada, pois não foi possível verificar a influência dos CQ no desempenho organizacional, contrariando o 
mencionado pela literatura. Um fator que pode explicar esses resultados, é que produtos com baixa qualidade (desde que estejam na moda), tenham seu espaço no mercado e, além disso, pode-se dizer que os produtos com algum defeito são comercializados como segunda linha no mercado varejista local (MONTEIRO, 2008). Fatores esses que talvez sejam percebidos pelos empresários como de maior impacto no desempenho do que acompanhar/controlar os custos da qualidade.

Além disso, outro ponto a ser destacado se refere à forma como os CQ são usados pelas empresas da amostra e a frequência de utilização dos mesmos. Foi verificado que $52,9 \%$ das empresas pesquisadas mensuram os CQ e, a maioria, calcula os custos de falhas internas e falhas externas. Esses resultados podem ser indício de que os CQ não são usados de forma efetiva nessas empresas, o que se reflete na rejeição da hipótese ligada à influência dos CQ no desempenho organizacional.

\section{Considerações Finais}

O estudo buscou verificar a influência dos fatores internos, estratégia, tecnologia e inovação, na ocorrência dos CQ, e ainda, a influência da ocorrência dos CQ no desempenho organizacional percebido de empresas pertencentes ao APL de confecções dos municípios de Maringá e Cianorte, no Estado do Paraná. A amostra de 121 empresas foi analisada por meio da técnica multivariada de modelagem de equações estruturais pelo método de estimação dos mínimos quadrados parciais (PLS) a fim de constatar a influência entre as variáveis estudadas.

Foi possível verificar por meio da modelagem de equações estruturais, que a estratégia de diferenciação possui um efeito de redução na ocorrência dos custos de falhas internas e externas, e ainda, a estratégia de liderança em custos incentiva que os custos com avaliação ocorram e restringe a ocorrência de custos com falhas tanto internas como externas.

Constatou-se que a adoção de tecnologia de informação auxilia a ocorrência de custos de prevenção e a reduzir custos de falhas internas, bem como, a utilização de tecnologia de produção reduz os custos com devoluções e substituições de produtos. A inovação dos produtos não apresentou efeito significativo sobre a ocorrência dos CQ.

Um achado impressionante é que a inovação dos processos apresentou um efeito negativo sobre os custos de falhas internas e externas, ou seja, devido a inovação e a consequente incerteza das tarefas, as empresas ficam mais suscetíveis para ocorrer custos com falhas.

A ocorrência dos custos da qualidade não possuiu influência sobre o desempenho organizacional. Tais achados podem ser em decorrência das características peculiares do setor de confecções. Uma vez que os produtos que não apresentarem qualidade suficiente podem ser vendidos muitas vezes como segunda linha, não resultando em total perda para estas organizações.

Reconhece-se, diante do exposto, que a presente pesquisa possui implicações. Sugerese que sindicatos, associações e entidades do setor de confecções proporcionem auxílio, principalmente às micro e pequenas empresas, no que se refere a inovação dos produtos de confecções a fim de que estas alcancem um melhor desempenho organizacional.

Esta pesquisa possui como limitações a amostra, que representa apenas $46,5 \%$ da população, assim não se podem generalizar os resultados, bem como, as variáveis estudadas, podendo outros fatores influenciar no âmbito empresarial além dos três fatores contingenciais internos que foram investigados nesta pesquisa.

Diante dos resultados, surgem novas oportunidades de estudo, como: [i] verificar a influência de outros fatores contingenciais nos CQ e no desempenho, como por exemplo, o porte, a estrutura, o estilo de liderança, a cultura organizacional e o ciclo de vida organizacional; 
[ii] estudos de casos a fim de investigar os fatores determinantes para a gestão dos CQ; [iii] estudos empíricos que analisem a institucionalização de práticas gerenciais de qualidade; [iv] além de estudos profundos que visem verificar a influência da gestão dos CQ no desempenho organizacional.

\section{Referências}

AGUIAR, Andson B.; FREZATTI, Fábio. Sistema de controle gerencial e contextos de processo de estratégia: contribuições da teoria da contingência. In: Congresso USP de Controladoria e Contabilidade. Anais...,2007.

AYRES, M. Cianorte aposta em conforto para vencer concorrência asiática. Maringá, Gazeta. (2012). Disponível em:

$<$ http://www.gazetadopovo.com.br/economia/especiais/potencialidades-parana/cianorteaposta-em-conforto-para-vencer-concorrencia-asiatica-6wtb8e 7kpgft4wk6d4ssuelou $>$. Acesso em 1 set. 2015.

BAINES, Annette; LANGFIELD-SMITH, Kim. Antecedents to management accounting change: a structural equation approach. Accounting, Organizations and Society, v. 28, n. 7, p. 675-698, 2003. DOI: 10.1016/S0361-3682(02)00102-2

BESSANT, John; TIDD, Joe. Inovação e empreendedorismo: administração. Porto Alegre: Bookman Editora, 2009.

BURNS, John; VAIVIO, Juhani. Management accounting change. Management Accounting Research, v. 12, n. 4, p. 389-402, 2001. DOI: 10.1006/mare.2001.0178

CADEZ, Simon; GUILDING, Chris. An exploratory investigation of an integrated contingency model of strategic management accounting. Accounting, Organizations and Society, v. 33, n. 7, p. 836-863, 2008. DOI: 10.1016/j.aos.2008.01.003

CHENHALL, Robert H. Management control systems design within its organizational context: findings from contingency-based research and directions for the future. Accounting, Organizations and Society, v. 28, n. 2, p. 127-168, 2003. DOI: 10.1016/S03613682(01)00027-7

COLLAZIOL, Elisandra. Custos da Qualidade: uma investigação da pratica e percepção empresarial. 2006. 138 p. Dissertação (Mestrado em Ciências Contábeis)-Programa de Pós Graduação em Ciências Contábeis, Universidade do Vale do Rio dos Sinos, São Leopoldo, 2006.

CORAL, Eliza. Avaliação e gerenciamento dos custos da não qualidade. 1996. $184 \mathrm{f}$. Dissertação (Mestrado em Engenharia de Produção)-Programa de Pós-Graduação em Engenharia de Produção,. Universidade Federal de Santa Catarina, Florianópolis, 1996.

COSTA, Ana Cristina Rodrigues da; ROCHA, Érico Rial Pinto da. Panorama da cadeia produtiva têxtil e de confecções e a questão da inovação. BNDES Setorial, Rio de Janeiro, n. 29, p. 159-202, 2009. 
CRUZ, Ana Paula Capuano. Estilo de liderança, sistema de controle gerencial e inovação tecnológica: papel dos sistemas de crenças, interativo, diagnóstico e de restrições. 2014. 168 f. Tese (Doutorado) - Programa de Pós-Graduação em Controladoria e Contabilidade, Universidade de São Paulo, São Paulo, 2014. DOI: 10.11606/T.12.2014.tde-02062014180339

DEMING, W. Edwards. Saia da crise. São Paulo: Futura, 2003.

DOBNI, C. Brooke. Measuring innovation culture in organizations: The development of a generalized innovation culture construct using exploratory factor analysis. European Journal of Innovation Management, v. 11, n. 4, p. 539-559, 2008. DOI:

$10.1108 / 14601060810911156$

DONALDSON, Lex. The contingency theory of organizations. Sage, 2001.

DONALDSON, Lex. Teoria da contingência estrutural. In S. Clegg, C. Hardy \& W. Nord (Orgs.). Handbook de Estudos Organizacionais. (v. 1, c. 3, pp. 105-133), São Paulo: Atlas, 2008.

DRAZIN, Robert; VAN DE VEN, Andrew H. Alternative forms of fit in contingency theory. Administrative Science Quarterly, p. 514-539, 1985. DOI: 10.2307/2392695

ESPEJO, Márcia Maria dos Santos Bortolocci. Perfil dos atributos do sistema orçamentário sob a perspectiva contingencial: uma abordagem multivariada. 2008. 216 f. Tese (Doutorado)-Programa de Pós-Graduação em Controladoria e Contabilidade, Universidade de São Paulo, São Paulo, 2008. DOI: 10.11606/T.12.2008.tde-30062008141909

FEIGENBAUM, Armand V. Controle da qualidade total. v. 4. São Paulo: Makron Books, 1994.

FIEP. Industriais e trabalhadores da confecção se unem para frear demissões. 2015. Disponível em: $<$ http://www.agenciafiep.com.br/noticia/industriais-e-trabalhadores-do-setortextil-e-da-confeccao-se-unem-para-superar-crise-e-frear-demissoes/ >. Acesso em 1 set. 2015.

GARVIN, D. A. Gerenciando a qualidade: a visão estratégica e competitiva. Rio de Janeiro: Qualitymark, 2002.

GORDON, Lawrence A.; NARAYANAN, Vadake K. Management accounting systems, perceived environmental uncertainty and organization structure: an empirical investigation. Accounting, Organizations and Society, v. 9, n. 1, p. 33-47, 1984. DOI: 10.1016/0361-3682(84)90028-X

GUERRA, A. R. Arranjos entre fatores situacionais e sistemas de contabilidade gerencial sob a ótica da teoria da contingência. 2007. Dissertação (Mestrado)-Programa de PósGraduação em Controladoria e Contabilidade, Universidade de São Paulo, São Paulo.

HAIR, Joseph F. et al. Análise multivariada de dados. Bookman Editora, 2009. 
HANSEN, D. R.; MOWEN, M. M. Gestão de custos. São Paulo: Cengage Learning, 2010.

HANSEN, Stephen C.; VAN DER STEDE, Wim A. Multiple facets of budgeting: an exploratory analysis. Management Accounting Research, v. 15, n. 4, p. 415-439, 2004. DOI: 10.1016/j.mar.2004.08.001

HYVÖNEN, Johanna. Strategy, performance measurement techniques and information technology of the firm and their links to organizational performance. Management Accounting Research, v. 18, n. 3, p. 343-366, 2007. DOI: 10.1016/j.mar.2007.02.001

JOHNSON, G.; SCHOLES, K.; WHITTINGTON, R. Explorando a estratégia corporativa. 7. ed. Porto Alegre: Bookman, 2007.

JUNQUEIRA, Emanuel R. Perfil do sistema de controle gerencial sob a perspectiva da teoria da contingência. 2010. 147 f. Tese (Doutorado)-Programa de Pós-Graduação em Controladoria e Contabilidade, Universidade de São Paulo, São Paulo, 2010. DOI: 10.11606/T.12.2010.tde-10052010-143511

JURAN, J. M. A qualidade desde o projeto: novos passos para o planejamento da qualidade em produtos e serviços. São Paulo: Cengage Learning, 2009.

JURAN, J. M.; GRYNA, F. M., Jr. Quality planning and analysis: from product development through usage. New Delhi: McGraw-Hill, 1970.

LANGFIELD-SMITH, Kim. Management control systems and strategy: a critical review. Accounting, Organizations and Society, v. 22, n. 2, p. 207-232, 1997. DOI: $10.1016 / \mathrm{S} 0361-3682(95) 00040-2$

MARÔCO, João. Análise de equações estruturais: fundamentos teóricos, software e aplicações. ReportNumber: Pêro Pinheiro, 2010.

NEITZKE, Ana Cláudia Afra. A coexistência de Apolo e Dionísio: influência da estratégia e do estilo de liderança no design e uso do orçamento sob a égide da teoria contingencial. 2015. 145 f. Dissertação (Mestrado)-Programa de Pós-Graduação em Ciências Contábeis, Universidade Federal do Paraná, Curitiba, 2015.

OLIVEIRA, Maria A.; CÂMARA, Márcia RG; BAPTISTA, Josil RV. O setor têxtilconfecções do Paraná e seus segmentos regionais especializados: 2000-2004. Revista de Economia, Editora UFPR, v. 33, n. 01, p. 83-115, 2007. DOI: $\underline{10.5380 / \text { re.v33i1.8548 }}$

PINTO, Leonardo José Seixas. Produção científica sobre custos da qualidade e da não qualidade no Brasil: um estudo bibliométrico nos principais livros, revistas acadêmicas e congressos na área de ciências contábeis. Registro Contábil, v. 3, n. 2, p. 60-74, 2012.

PORTER, Michael E. O que é estratégia. Harvard Business Review, v. 74, n. 6, p. 61-78, 1996. 
PORTER, M. E. Estratégia competitiva: técnicas para análise de indústrias e da concorrência. 2. ed. Rio de Janeiro: Elsevier, 2004.

RINGLE, Christian M.; DA SILVA, Dirceu; BIDO, Diógenes de Souza. Modelagem de equações estruturais com utilização do SmartPLS. REMark, v. 13, n. 2, p. 54, 2014. DOI: 10.5585/remark.v13i2.2717

ROBLES JUNIOR, Antonio. Custos da qualidade: aspectos econômicos da gestão da qualidade e da gestão ambiental. 2. ed. São Paulo: Atlas, 2003.

SÁ, V. M. R. de. Custo da qualidade nas indústrias de transformação de Pernambuco. 2003. 108 f. Dissertação (Mestrado em Engenharia de Produção)-Universidade Federal de Pernambuco, Recife, 2003.

SAKURAI, M. Gerenciamento integrado de custos. São Paulo: Atlas, 1997.

SERRÃO, M. A. dos S. Avaliação para o desenvolvimento de competências de liderança e inovação numa empresa de TI: um estudo experimental. 2009. 44 f. Dissertação (Mestrado em Psicologia dos Recursos Humanos)- Universidade de Lisboa, Lisboa, Portugal, 2009.

SMARTPLS. Standardized Root Mean Square Residual (SRMR).2016. Disponível em: $<$ http://www.smartpls.de/documentation/srmr $>$. Acesso em 22 jan. 2016.

TIDD, J.; BESSANT, J.; PAVITT, K. Gestão da inovação. Porto Alegre: Bookman, 2008.

TIGRE, P. B. Gestão da inovação: a economia da tecnologia do Brasil. Rio de Janeiro: Elsevier, 2006.

VAN DE VEN, Andrew H. Central problems in the management of innovation. Management Science, v. 32, n. 5, p. 590-607, 1986. DOI: $\underline{10.1287 / \mathrm{mnsc} .32 .5 .590}$ 
